\title{
Software to predict the right dose for vancomycin: the risk of 'pseudo-precision' in a clinical environment
}

\author{
Koos Dijkstra ${ }^{1}$, Agnes Veldkamp ${ }^{1}$, Elske Sieswerda ${ }^{1}$, and Michiel van Agtmael $^{2}$ \\ ${ }^{1}$ Affiliation not available \\ ${ }^{2}$ Amsterdam UMC - Locatie VUMC
}

June 25, 2020

\section{A commentary on: Personalised dosing of vancomycin: a prospec- tive and retrospective comparative quasi-experimental study by Luqman Vali et al.}

Vancomycin is frequently used to treat Staphylococcal infections resistant to beta-lactam antibiotics. Overexposure to vancomycin increases risk of nephrotoxicity while underexposure can lead to therapeutic failure. This narrow therapeutic window makes it necessary to measure vancomycin serum concentrations aiming for an effective and justifiable dose. Often mathematical software is used to calculate a pharmacokinetic (PK) / pharmacodynamic $(\mathrm{PD})$ target and predict the right vancomycin dosage.

In this journal, Vali et al. compared the prospective results of a bedside Bayesian-guided software package (DoseMeRx) to guide vancomycin dosing with retrospective results of vancomycin-dosing with a standard algorithmic approach. Bedside Bayesian-guided dosing resulted in significantly more area under the curves (AUC) measured within the target range $\left(350-450 \mathrm{mg} / \mathrm{L}^{*} \mathrm{~h}\right)$ and in a significantly higher percentage of time in the acceptable range in comparison with the standard algorithmic approach.

Unfortunately, the authors were not able to relate their findings to clinical outcomes such as clinical cure or mortality, possibly due to insufficient power. Still, PK/PD modelling is the cornerstone in calculating vancomycin dosages and user friendly bedside software can increase the availability and integration of these models in clinical practice. However, the precision of the dose calculations based on pharmacokinetic and pharmacodynamic modelling can be misleading and should not be used to determine the optimal dose without further considerations.

Several factors impact the interpretation of individual dose calculations of antibiotics based on PK/PD modelling: the PK/PD-index of the antibiotic that is used to predict the efficacy; clinical and pharmacological factors that influence PK and the accuracy of minimal inhibitory concentration (MIC) of the causative pathogen. We will discuss these three factors and elaborate on their influence on dose calculations.

Several studies aimed to establish the dose-response relation of vancomycin. Generally accepted vancomycin $\mathrm{PK} / \mathrm{PD}$ targets are an $\mathrm{AUC} / \mathrm{MIC}>400$ or a trough serum or plasma level $\left(\mathrm{C}_{\text {trough }}\right) / \mathrm{MIC}>10-15$. Using the AUC/MIC as predicting parameter requires the use of specialized software to calculate the AUC on intermittent dosing regimens, but is generally accepted as the most appropriate PK/PD-index of vancomycin. A recent meta-analysis of several cohort studies showed that AUC/MIC guided dosing resulted in a lower incidence acute kidney injury (AKI) in comparison to trough level dosing with an odds ratio of 0.68 (95\% CI: 0.46 - 0.99). However, included studies were small, mostly retrospective and the effect of PK/PD-based dosing on AKI and other clinical outcomes therefore need to be confirmed in larger studies. 
Experiments with novel in-vitro models, such as two-compartment hollow fiber models, could add valuable information to the currently available evidence on the optimal PK/PD index of vancomycin. However, as far as we are aware, studies with these novel in-vitro models with vancomycin have not yet been reported.

Tissue penetration is another major determinant in applying PK/PD calculations in clinical practice. The extent of tissue penetration depends on the solubility of the compound in fat (or octanol - the $\log P$ ), the acid dissociation constant ( $\mathrm{pKa}$ ), the extent of protein binding, the molecular mass and the affinity to certain transmembrane transporters, such as P-glycoprotein. It could be questionable if serum or plasma concentrations are a representative factor in the treatment outcome when the tissue penetration of an antibiotic is low, while the infection is mainly situated in tissue. A good example is the high molecular mass of vancomycin and therefore low penetration characteristics.

Microdialysis, has delivered promising results to quantify the tissue penetration of antibiotics. With microdialysis, a small dialysis tube is placed in the tissue of interest. Due to diffusion of the antibiotic from the extracellular fluid into the dialysis fluid, the antibiotic concentration can be measured in the dialysate. Microdialysis can help to extrapolate the PK/PD index to infections in tissue either by computing serumtissue ratios or by building multiple-compartment PK models. However, a complicating aspect is the large interindividual variation in tissue concentrations. Studies on microdialysis with vancomycin have shown large variation in interstitial fluid concentrations which could not be explained by patient characteristics. In addition, the penetration of vancomycin in the central nervous system (CNS) is also highly variable. For CNS infections, one should consider to administer vancomycin intrathecally or switch to a different antibiotic with proven acceptable penetration in the CNS, such as linezolid. Overall, vancomycin tissue penetration is relatively low but some high unexplained variability is observed. Overall, microdialysis and hollow fiber models are potentially useful techniques in gaining more insight in the relation between serum concentrations, tissue concentrations and efficacy. Yet, clinical evidence for their use is still limited.

Beside the optimal PK/PD target and extent of tissue penetration, the way of dosing is also of importance. Continuous vancomycin administration is increasing in clinical practice, making sampling easy as the concentration measured does not depend on the time of sampling. Furthermore calculation of the $\mathrm{AUC}_{0-24 \mathrm{~h}}$ $\left(\mathrm{mg} / \mathrm{L}^{*} \mathrm{~h}\right)$ of vancomycin on continuous infusion is simple: multiply the measured concentration (in $\mathrm{mg} / \mathrm{L}$ ) by 24 . In a meta-analysis the incidence of nephrotoxicity was found lower in patients treated with continuous vancomycin vs. patients treated with intermittent vancomycin, with no difference in treatment failure or mortality. A limiting practical factor in applying continuous vancomycin is the availability of one dedicated intravenous (IV) catheter or lumen, since vancomycin is incompatible with many other medicines and IV fluids. In the paper by Vali et al. we missed vancomycin continuous infusion simulations to collect evidence for this upcoming method of administration.

A final major determinant of PK/PD modelling is the MIC. The MIC is defined as the lowest concentration that inhibits growth of the isolated micro-organism. The golden standard for MIC testing is broth microdilution. However, many clinical microbiological laboratories use automated systems or antimicrobial gradient strips for their first-line antimicrobial susceptibility testing (AST). These methods are rarely precise enough to be used to calculate the AUC/MIC or $\mathrm{C}_{\text {trough }} / \mathrm{MIC}$. Therefore, Mouton et al. suggested to include the epidemiological cut-off value (ECOFF) when interpreting MIC results for target attainment calculations. The ECOFF is determined as the highest MIC of the bacterial species of interest without acquired resistance, i.e. the wildtype population. Using the ECOFF instead of the MIC, however could add potential risks of overdosing of an antibiotic for the wildtype population of a micro-organism with broad MIC ranges. For example, the suggested ECOFF of $S$. aureus for vancomycin is $2 \mathrm{mg} / \mathrm{L}$, which would indicate that the target $\mathrm{AUC}_{0-24 \mathrm{~h}}$ should be above $800 \mathrm{mg} / \mathrm{L}^{*} \mathrm{~h}(\mathrm{AUC} / \mathrm{MIC}>400)$. Such high AUCs increase the risk of nephrotoxicity and should not be applied in regular care. PK/PD-based modelling with accurate MIC testing therefore seems of importance to decrease risk of overdosing.

In conclusion, the use of software programs can facilitate the measurement of these targets and the prediction of the right dose of vancomycin. The prescriber and pharmacist should, however, always be aware of the 'pseudo- precision' of those calculations: many other factors influence the efficacy and toxicity of vancomycin 
besides the trough concentration or the AUC.

\section{References}

1. Rybak MJ, Lomaestro BM, Rotschafer JC, et al. Therapeutic Monitoring of Vancomycin in Adults: Summary of Consensus Recommendations from the American Society of Health-System Pharmacists, the Infectious Diseases Society of America, and the Society of Infectious Diseases Pharmacists. Pharmacotherapy. 2009;29(11):1275-1279. doi:10.1592/phco.29.11.1275

2. Aljefri DM, Avedissian SN, Rhodes NJ, Postelnick MJ, Nguyen K, Scheetz MH. Vancomycin Area Under the Curve and Acute Kidney Injury: A Meta-analysis. Clin Infect Dis. 2019;69(11):1881-1887. doi:10.1093/cid/ciz051

3. Rizk ML, Bhavnani SM, Drusano G, et al. Considerations for Dose Selection and Clinical Pharmacokinetics/Pharmacodynamics for the Development of Antibacterial Agents. Antimicrob Agents Ch. 2019;63(5). doi:10.1128/aac.02309-18

4. European Medicines Agency. Guideline on the use of pharmacokinetics and pharmacodynamics in the development of antimicrobial medicinal products. Published online 2017.

5. Mouton JW, Muller AE, Canton R, Giske CG, Kahlmeter G, Turnidge J. MIC-based dose adjustment: facts and fables. J Antimicrob Chemoth. 2017;73(3):564-568. doi:10.1093/jac/dkx427

6. Jager NGL, Hest RM van, Lipman J, Roberts JA, Cotta MO. Antibiotic exposure at the site of infection: principles and assessment of tissue penetration. Expert Rev Clin Phar. 2019;12(7):1-12. doi:10.1080/17512433.2019.1621161

7. Abraham J, Sinnollareddy MG, Roberts MS, et al. Plasma and interstitial fluid population pharmacokinetics of vancomycin in critically ill patients with sepsis. Int J Antimicrob Ag. 2019;53(2):137-142. doi:10.1016/j.ijantimicag.2018.09.021

8. Beach JE, Perrott J, Turgeon RD, Ensom MHH. Penetration of Vancomycin into the Cerebrospinal Fluid: A Systematic Review. Clin Pharmacokinet. 2017;56(12):1479-1490. doi:10.1007/s40262-017-0548-y

9. Hamada Y, Kuti JL, Nicolau DP. Vancomycin serum concentrations do not adequately predict tissue exposure in diabetic patients with mild to moderate limb infections. J Antimicrob Chemoth. 2015;70(7):20642067. doi:10.1093/jac/dkv074

10. Hao J-J, Chen H, Zhou J-X. Continuous versus intermittent infusion of vancomycin in adult patients: A systematic review and meta-analysis. Int $J$ Antimicrob Ag. 2016;47(1):28-35. doi:10.1016/j.ijantimicag.2015.10.019 